\title{
Primary synchronous small and non-small cell lung cancer in the same lung lobe: $A$ case report
}

\author{
Anton Dzian', Ivan Fúčela', Zdenko Hut'ka², Peter Szépe² \\ 1 Thoracic Surgery Clinic, Jessenius Faculty of Medicine in Martin, Comenius University, Martin \\ 2Department of Pathology, Jessenius Faculty of Medicine in Martin, Comenius University, Martin, Slovakia
}

\begin{abstract}
Synchronous multiple primary lung cancer (SMPLC) means tumours present at the same time, which are separate and have different histology. We present the case of a 66 -year-old patient with a combination of small-cell lung carcinoma (SCLC) with adenocarcinoma in the same lobe with metastasis of SCLC in the mediastinal lymph node. This is a rare case. We performed a standard left upper video-assisted thoracoscopic lobectomy with mediastinal lymphadenectomy and adjuvant therapy targeted to SCLC was administered. Despite advances in imaging methods and diagnostic procedures in pneumology a diagnosis of SMPLC is often discovered only intraoperatively or accidentally during the histological examination. Actually, no guidelines exist for the treatment protocol of such cases. Multiple methods of management of SMPLC, mainly complete anatomical resection with lymphadenectomy combined with chemotherapy or radiotherapy should be adopted according to the histologic types, staging and molecular testing of the tumours. These rare cases of SMPLC require individual treatment and multidisciplinary approach.
\end{abstract}

Corresponding author: Anton Dzian, Thoracic Surgery Clinic, Jessenius Faculty of Medicine in Martin, Comenius University, Kollárova 2, 03659 Martin, Slovakia. Tel. +421.43.4203 418. E-mail: anton.dzian@gmail.com

Key words: Synchronous lung cancer; adenocarcinoma; small-cell lung cancer; VATS lobectomy.

Contributions: AD collected all clinical data. All authors participated in the preparation of the manuscript and read and approved the final version.

Conflict of interest: The authors declare no conflict of interest.

Received for publication: 21 November 2016

Accepted for publication: 5 November 2017

(C) Copyright A. Dzian et al., 2017

Tipografia PI-ME Editrice, Italy

Monaldi Archives for Chest Disease 2017; 87:797

doi: 10.4081/monaldi.2017.797

This article is distributed under the terms of the Creative Commons Attribution Noncommercial License (by-nc 4.0) which permits any noncommercial use, distribution, and reproduction in any medium, provided the original author(s) and source are credited.

\section{Introduction}

Synchronous multiple primary lung cancer (SMPLC) is defined by Martini and Melamed as tumours that are present at the same time. They must be separate and the histology must be different [1]. SMPLC is a rare entity with an incidence rate ranging from $1 \%$ to $7 \%$ [2]. The coexistence of small and non-small cell carcinoma has been reported in a very small fraction of cases $[3,4]$. We present a case of multiple synchronous primary lung malignancies of different histological types (adenocarcinoma and small-cell lung cancer (SCLC)) in the same lung lobe with metastasis SCLC in the mediastinal lymph node.

\section{Case Report}

In February 2015, a 66-year-old man was admitted to our hospital with suspected lung cancer because of an abnormal finding in the left lung on a chest X-ray after healing pneumonia. He had smoked 30 cigarettes per day for the past 58 years. He had no family history of lung cancer and no respiratory symptoms. Physical examination revealed no abnormalities.

Contrast-enhanced chest CT images indicated peripheral tumours in the left upper lobe with $1.5 \mathrm{~cm}$ diameter (S3- anterior segment) and 2.8 cm diameter (S1, 2 - apical posterior segment) (Figure 1). The aortopulmonary window lymph node of the mediastinum was described as enlarged. Positron emission tomography with 2-deoxy-2-[fluorine-18] fluoro- D-glucose integrated with computed tomography (18F-FDG PET/CT) showed that the two lung masses and the aortopulmonary window lymph node were hypermetabolic. Complete PET/CT scan was negative for distant metastases.

Laboratory findings were within normal limits. Tumour marker such as carcinoembryonic antigen (CEA) was mildly elevated, neuron-specific enolase (NSE), cytokeratin fragment (CYFRA 21-1) and chromogranin A were normal. Repeated sputum cytology showed no malignant cells. Bronchoscopic examination showed no abnormal findings within the visible area. Bronchoalveolar lavage and brushing cytologic studies were negative. CT-guided percutaneous needle aspiration of the left upper lobe masses was negative. The spirometry results were quite satisfactory. Preoperative respiratory values were forced expiratory volume in the $1^{\text {st }}$ second (FEV1) 101\% (4.01 L), diffusion lung carbon monoxide (DLCO) $96 \%(10.6 \mathrm{mmol} / \mathrm{kPa} / \mathrm{min})$. Predicted postoperative values (ppo) were: ppoFEV1 85\% (3.37 L), ppoDLC0 81\% (9.1 $\mathrm{mmol} / \mathrm{kPa} / \mathrm{min}$ ).

Because preoperative diagnosis of the two lesions and the aortopulmonary window lymph node was not possible, the patient underwent standard left upper video-assisted thoracoscopic VATS lobectomy with mediastinal lymphadenectomy from stations 5, 6, 7, 9 and 10 . The pa- 
tient's postoperative course was uneventful and he was discharged on the $4^{\text {th }}$ post-operative day.

Pathologic examination of the left upper lobe revealed two lesions. The $S 1,2$ tumour was confirmed to be a moderately differentiated lung adenocarcinoma (Figure $2 \mathrm{~A}, \mathrm{~B}$ ), which was positive for cytokeratin (CK) 7, thyroid transcription factor-1 (TTF-1) and negative for CK20 and CDX-2 expression. The tumour located in the anterior segment (S3) of the left upper lobe showed histologically as high grade small-

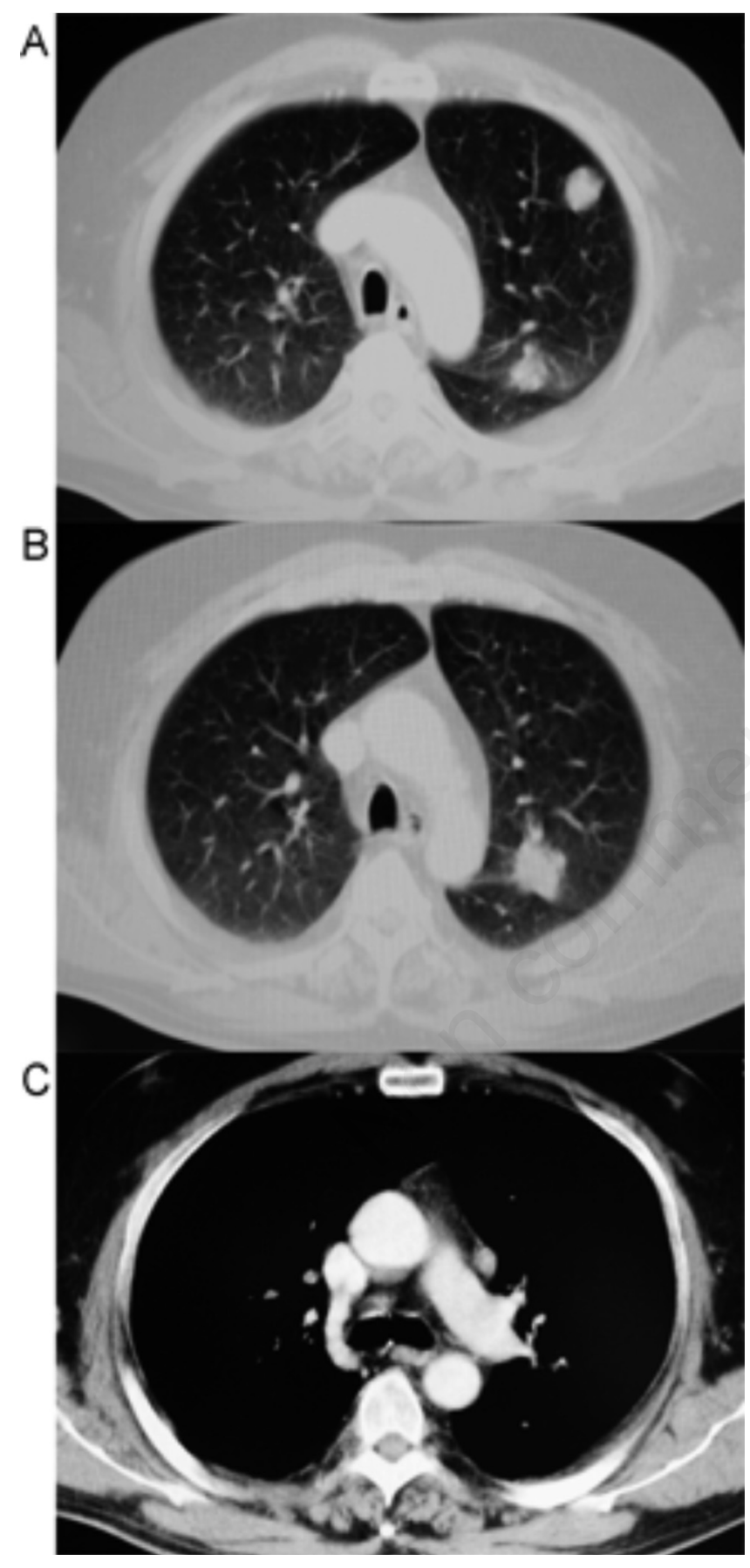

Figure 1. Chest CT. A) The $1.5 \mathrm{~cm}$ diameter peripheral tumor in the left upper lobe (S3 - anterior segment); B) $2.8 \mathrm{~cm}$ (S1,2 - apical posterior segment) diameter peripheral tumor in the left upper lobe; C) enlarged aortopulmonary window lymph node of the mediastinum. cell lung cancer (Figure 2 C,D). Immunohistochemically it was positive for CD56, CK8/18 and negative for TTF-1, CK7, CK20, p63, CK5/6, vimentin, desmin. The surgical margins and hilar lymph nodes were free of disease. One aortopulmonary window lymph node was positive for SCLC, the other mediastinal lymph nodes were negative. The final diagnosis with regards to postoperative clinical staging was stage IA (T1aN0M0) for adenocarcinoma and IIIA (T1bN2M0) for the SCLC. Molecular testing for epidermal growth factor receptor (EGFR) and anaplastic lymphoma kinase (ALK) gene mutations was performed in the adenocarcinoma tumor. EGFR mutations in exon 18, 19, 20 and 21 were not identified. The test for $2 \mathrm{p} 23 / \mathrm{ALK}$ rearrangement was negative.

The patient's postoperative course was uncomplicated and the patient was released from the hospital five days after the operation. Four cycles of combination chemotherapy with etoposide and carboplatin and thoracic radiotherapy at a dose of $64 \mathrm{~Gy}$ were administered. He was followed as an outpatient without complications. Prophylactic cranial irradiation was indicated, but the patient refused despite guidance. Sixteen months after the operation the patient underwent a PET/CT scan which found positive liver tumours histologised as metastasis of small-cell lung carcinoma, the lungs and mediastinum were negative. The patient died 19 months after the operation through the progression of the malignant disease.

\section{Discussion}

Synchronous multiple primary lung cancers have been revealed more frequently over the last years due to diagnostic improvements in detecting small lesions and they cause difficulties in determining the aetiology of multiple malignant lung lesions. In cases in which more than one tumour is detected radiologically, the possibility of a synchronous tumour or a metastasis of the primary tumour must always be kept in mind and histopathologic diagnosis should be done for both tumours [5]. The lung masses of small and non-small cell carcinoma cannot be discriminated by imaging studies because they usually have similar morphology on CT and the same SUV on 18F-FDG PET/CT [6]. Using bronchoscopy separate biopsies should be taken from both lesions when available. When mediastinal lymphadenopathy is present, endobronchial ultrasound guided fine needle aspiration biopsy of mediastinal nodes for histological sampling is preferred. An invasive histopathologic examination such as CT-guided lung biopsy, mediastinoscopy or video-assisted thoracoscopic surgery (VATS), is considered necessary in the case where two abnormal nodules are obviously separated in the same lobe and mediastinal lymphadenopathy is present on chest CT. Despite the latest advances in diagnostic technologies, SMPLC diagnosis is often only a hypothesis preoperatively while- in a high percentage of case, SMPLC are discovered only intraoperatively or accidentally during histological examination [7].

Synchronous multiple tumors of histologically different cell types should be considered as separate primary lung cancers and should be staged separately [8]. The definitive pathological diagnosis of SMPLC is based on histological or cytological examination, with usage of modern immunohistochemistry and molecular techniques. Molecular testing for EGFR and ALK gene mutations in lung adenocarcinoma is critical for possible target therapy.

Multiple methods of management of SMPLC, mainly resection combined with chemotherapy or radiotherapy should be adopted according to the histologic types, staging and molecular testing of the tumours. Complete anatomical resection and lung parenchymal preservation resection in functionally limited patients, with lymphadenectomy in both, should be performed if feasible. VATS lobectomy is actually safe and may achieve similar long-term survival times as the open surgery ap- 


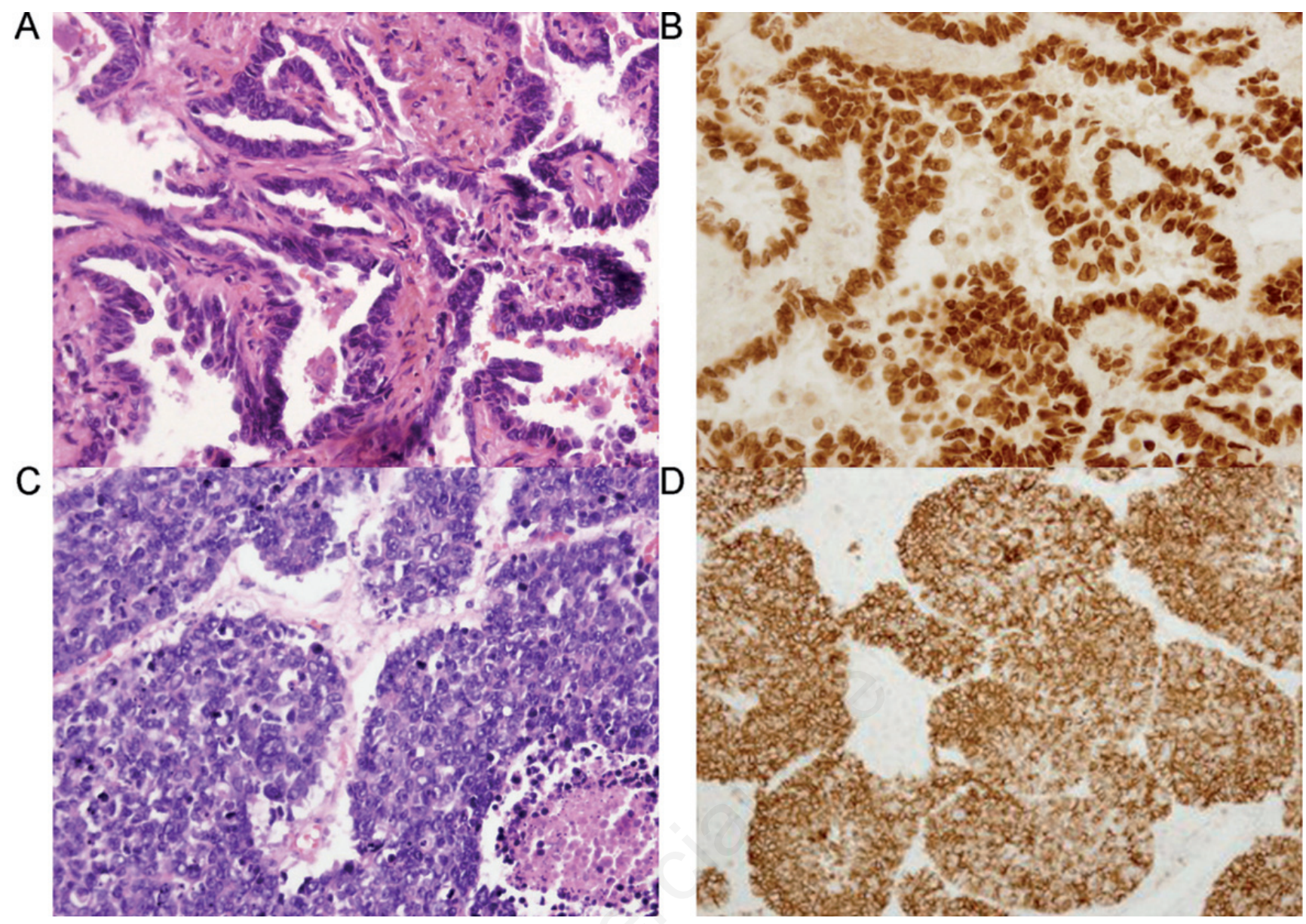

Figure 2. A) Adenocarcinoma, hematoxylin and eosinstain, original magnification $\times 20$; B) adenocarcinoma, thyroid transcription factor-1 $($ TTF-1) immunostaining, original magnification $\times 20$; C) small cell lung cancer (SCLC), hematoxylin and eosinstain, original magnification $\times 20$; D) SCLC, CD 56 immunostaining, original magnification $\times 10$.

proach. An aggressive surgical approach to patients with apparent SMPLC can result in survival that is comparable with patients with single lung cancers of similar stage [9]. In the current case, the preoperative diagnostic methods have not resulted in histologically verified tumours. Tumours were resectable and the patient was indicated for resection treatment. Adjuvant oncologic therapy should be indicated for the high grade and prognostic worse type of SMPLC. In our case therapy was targeted to small-cell lung cancer. According to the European Society for Medical Oncology Clinical Practice Guidelines for SCLC treatment all patients with T1-4, N0-3 M0 tumours who are in good performance status should be treated with concurrent chemotherapy and thoracic radiotherapy. All SCLC patients responding to first-line treatment should be evaluated for prophylactic cranial irradiation [10].

Subsequent studies of SMPLC over the last years have been difficult to interpret due to the small overall numbers of cases and insufficient information regarding tumour characteristics and stage [9]. A study with a larger number of patients published by Trousse et al. reported 3 - and 5 -year survival at a rate of $48 \%$ and $34 \%$, respectively [11]. Riquet et al. reported 3- and 5-year survival at a rate of $40 \%$ and $26 \%$, respectively [12]. Patients with SMPLC in a study by Finley et al. with stages IA and IB who were surgically treated have a $74 \%$ and $65 \% 3$-year overall survival, respectively [9].

\section{Conclusions}

The combination of small-cell lung carcinoma with adenocarcinoma in the same lobe, as in the present case, is rare. Actually, no guidelines exist for the treatment protocol of such cases. These cases require individual treatment and multidisciplinary approach.

\section{References}

1. Martini N, Melamed M. Multiple primary lung cancer. J Thorac Cardiovasc Surg 1975;70:606-12.

2. Ferguson MK, DeMeester TR, DesLauriers J, et al. Diagnosis and management of synchronous lung cancers. J Thorac Cardiovasc Surg 1985;89:378-85.

3. Hiraki A, Ueoka H, Yoshino T, et al. Synchronous primary lung cancer presenting with small cell carcinoma and non-small cell carcinoma: diagnosis and treatment. Oncol Rep 1999;6:75-155.

4. Gogakos AS, Paliouras D, Rallis T, et al. Double primary non-small cell lung cancer with synchronous small cell lung cancer N2 nodes: a case report. Ann Transl Med 2005;3:157. 
5. Pierard P, Vermylen P, Bosschaerts T, et al. Synchronous roentgenographically occult lung carcinoma in patients with resectable primary lung cancer. Chest 2000;117:779-85.

6. Park KY, Koh JS, Choe DW, et al. Synchronous small and non-small cell lung cancer in a patient with previous tuberculosis. Intern Med 2007;46:1677-8.

7. Scialpi M, Franzini C, Piscioli I, et al. Synchronous primary lung cancer. Critical review of diagnostic criteria. Ann Ital Chir 2013;84:33-40.

8. Akgül AG, Batirel HF, Yildizeli B, et al. Synchronous lung tumors. Turk Resp J 2004;5:128-30.
9. Finley DJ, Yoshizawa A, Travis W, et al. Predictors of outcomes after surgical treatment of synchronous primary lung cancers. J Thorac Oncol 2010;5:197-205.

10. Früh M, De Ruysscher D, Popat S, et al. Small-cell lung cancer (SCLC): ESMO Clinical Practice Guidelines for diagnosis, treatment and follow-up. Ann Oncol 2013;24(Suppl 6):99-105.

11. Trousse D, Barlesi F, Loundou A, et al. Synchronous multiple primary lung cancer: an increasing clinical occurrence requiring multidisciplinary management. J Thorac Cardiovasc Surg 2007 133:1193-200.

12. Riquet M, Cazes A, Pfeuty K, et al. Multiple lung cancers prognosis: what about histology? Ann Thorac Surg 2008;86:921-6. 